\title{
Integration on a microchip: A glimpse into the future of optical coherence tomography
}

\author{
B. Imran Akca ${ }^{a}, *$, Lantian Chang ${ }^{b}$, and Gunay Yurtsever \\ ${ }^{a}$ LaserLab, Department of Physics and Astronomy, VU University Amsterdam, Amsterdam, The Netherlands \\ ${ }^{\mathrm{b}}$ Optical Sciences Group, University of Twente, Mesa + Institute, Enschede, The Netherlands \\ ${ }^{c}$ IMEC, Leuven, Belgium \\ *Corresponding author. e-mail address: b.i.avci@vu.nl
}

\section{Contents}

1. Introduction 2

2. Essential OCT parameters 4

3. Passive on-chip OCT components 6

3.1 Optical waveguides 6

3.2 Interferometer 8

$\begin{array}{ll}3.3 \text { Spectrometer } & 18\end{array}$

$\begin{array}{ll}3.4 \text { Reference arm } & 21\end{array}$

3.5 Sample arm 25

4. OCT imaging with a partially-integrated SD-OCT system 27

5. Conclusions and outlook 30

References 30

\section{Abstract}

Optical coherence tomography (OCT) is a noninvasive, three-dimensional imaging technique that offers close-to-histology-level image quality. Based on broadband spectral interferometry, OCT has enabled clinical applications ranging from ophthalmology to cardiology that revolutionized in vivo medical diagnostics. Considering the size and cost of a commercial OCT system, it is essential to investigate different approaches for realizing a compact and low-cost OCT system in order to make it accessible to a significantly larger group of applications and users. Exploiting integrated optics, several central components of an OCT system have been assembled on a microchip so far. Silicon based designs have the advantage of complementary metal-oxide-semiconductor (CMOS) compatible high-volume production while indium phosphate (InP) based designs have the possibility of monolithic integration of the light source and the detector with other components. In this chapter, the design considerations, theoretical analysis and experimental results of the passive integrated optical components of an on-chip OCT system including interferometer, spectrometer, reference arm, and sample arm will be presented. Active components (light source and detectors) are beyond the scope of this chapter. 
Keywords: Optical coherence tomography (OCT), Integrated optics, Medical imaging, Optical waveguides, Thin film semiconductors, Spectrometers, Miniaturized

\section{Introduction}

Optical coherence tomography (OCT) is a noninvasive optical technique for high-resolution cross-sectional imaging of, among other things, biological tissue, with many applications in clinical medicine. OCT is an extension of optical low-coherence domain reflectometry, which is a one-dimensional optical ranging technique based on low-coherence interferometry. It was first developed for measuring reflections in fiber optics and optoelectronic devices (Youngquist, Carr, \& Davies, 1987); in the following years the transverse scanning capability enabled cross-sectional imaging, i.e., OCT (Huang et al., 1991). The first OCT images were of the human retina and coronary arteries (Huang et al., 1991). To date, the most significant clinical impact of OCT has been in the field of ophthalmology. This is also why OCT is considered as the golden standard for cross-sectional retina imaging in ophthalmology. In the past decade, applications in OCT have expanded into other medical fields such as gastroenterology (Tearney et al., 1997a), gynecology (Pitris et al., 1999), pulmonology (Pitris et al., 1998), urology (Tearney et al., 1997b), cardiology (Brezinski et al., 1996), and oncology (Jung et al., 2005). It has a great potential in diagnosing and monitoring of cancerous tissue due to its very high resolution. Besides medical applications, OCT started gaining visibility in different application areas; such as agriculture. There is a strong demand in developing inexpensive and reliable technologies for non-destructive screening of internal defects and rots. Additionally, seed inspection and quality control are becoming crucial in order to preserve genetic pool of different crops in seed banks for future breeding requirements. OCT has been used in detecting infected seeds of several fruits and vegetables (Clements et al., 2004; Lee, Lee, Kim, Jung, \& Kim, 2011; Verboven et al., 2013). According to researchers, the main drawbacks of using OCT in agricultural applications are the complexity, sensitivity and high price of the equipment.

The working principle of OCT is similar to that of ultrasound; however, instead of sound waves OCT uses the reflection of light. Essentially, there are two main types of OCT systems; time-domain (TD) OCT and frequencydomain (FD) OCT. Although the operating mechanisms of TD-OCT and FD-OCT systems differ, the basic principle is the same. Both methods 
measure the interference of light reflected from the specimen with light reflected from a reference mirror. FD-OCT uses a fixed-position reference mirror, and resolves the interference as a function of light frequency. The depth structure can be reconstructed by observing the frequency of interference fringes, which translates into reflection profile over the path length difference. This results in a superior sensitivity and speed performance of FD-OCT systems over TD-OCT systems. There are two types of FDOCT systems: "spectral-domain OCT" (SD-OCT) using a broadband light source and a spectrometer and "swept-source OCT" (SS-OCT) using a rapidly wavelength-tuned laser. Both systems typically contain a combination of fiber and free-space components, which add to the instrument size and cost, affect its mechanical stability, and therefore require active alignment.

Integrated systems hold promise for wider deployment of OCT technology and open new fields of applications. On-chip OCT systems have recently become quite popular for their high potential in overcoming the size and cost problems of the bulky OCT systems. In addition to its low-cost and small footprint, this approach provides mechanical stability due to its monolithic and alignment-free construction. Moreover, integrated optics can enhance the performance of OCT by, for example, parallelization (Bourquin et al., 2005; Bourquin, Seitz, \& Salathé, 2001) of OCT devices on a chip. So far, the interferometer, spectrometer, scanning reference arm, light source, and the detector of an on-chip OCT system have been realized in different semiconductor material platforms (Akca, 2016a, 2016b; Akca, Chang, et al., 2012; Akca et al., 2012, 2013; Akca, Weiss, Coumans, \& van Leeuwen, 2017; Chang et al., 2016; Choi et al., 2008; Culemann, Knuettel, \& Voges, 2000; Jiao et al., 2012; Margallo-Balbas, Pandraud, \& French, 2008; Nguyen et al., 2011, 2012; Tilma et al., 2012; van Leeuwen et al., 2018; Yurtsever et al., 2014; Yurtsever, Weiss, Kalkman, van Leeuwen, \& Baets, 2014). Silicon based designs have the advantage of complementary metal-oxide-semiconductor (CMOS) compatible high-volume production and relatively low losses; however, they can only be used for passive components. On the other hand, compound semiconductor (e.g., InP) based designs have the possibility of monolithic integration of the light source and the detector with passive components, however they are CMOS incompatible and have relatively high loss values. For a fully integrated OCT system, hybrid integration of compound semiconductors with Si platforms could be an alternative way to pursue (Doerr, 2015; Thomson et al., 2016). In the following sections, the design considerations and experimental results of passive on-chip SD-OCT components will be discussed. 


\section{Essential OCT parameters}

The essential parameters that determine the imaging quality of SD-OCT systems are center wavelength, axial resolution, maximum imaging depth, signal-to-noise ratio (SNR), and sensitivity roll-off in depth. First, the OCT imaging depth is limited by the amount of scattering (higher at shorter wavelengths) and absorption (higher at longer wavelengths) in biological tissue. Therefore, the OCT imaging depth depends on the choice of center wavelength of the OCT system. Tissue is a non-homogeneous medium, which is constituted of cells maintained in a lattice. As a consequence light is incident on tissue is scattered off these structures. Additionally, tissue can absorb light by chromophore molecules that it contains (Andersen, Jørgensen, Thrane, Tycho, \& Yura, 2008). Therefore, the interaction of light with biological tissue occurs through scattering and absorption. The overall effect of scattering and absorption processes is the attenuation of ballistic light in depth, which results in reduction of the imaging depth and contrast, and resolution of the OCT system. Only backscattered photons from a target layer selected by the coherence gate of the light source contribute to the useful depth information, whereas bulk backscattered photons increase the noise in the OCT signal (Andersen et al., 2008). Mainly two common OCT wavelengths $\left(\lambda_{c}\right)$ are used in most of the commercial OCT systems; $800 \mathrm{~nm}$ for high-resolution retina imaging and $1300 \mathrm{~m}$ for dermal imaging where scattering is lower.

Second, the axial resolution of an SD-OCT system is determined by the center wavelength and effective bandwidth of the light that is detected. It depends on both, the bandwidth of the light source and the bandwidth of the spectrometer. If the spectrum of the light source, as measured with the spectrometer, has a Gaussian envelope with full width at half maximum (FWHM) $\Delta \lambda_{F W H M}$, the axial (depth) resolution $\Delta z$ is given by Swanson et al. (1992)

$$
\Delta z=\frac{2 \ln 2}{\pi n}\left(\frac{\lambda^{2}}{\Delta \lambda_{F W H M}}\right)
$$

where $n$ is the (group) refractive index of the imaged tissue and $\lambda$ is the center wavelength of the source.

Third, the maximum imaging depth $z_{\max }$ in SD-OCT is determined by the spectral sampling interval ( $\delta k, k$ is the wavenumber). From Nyquists' sampling theorem, the spectral sampling at $\delta k$ spacing leads to a maximum 
path length of $1 /(2 \delta k)$. However, since every path length corresponds to half the depth (light travels back and forth to the detector), the imaging depth becomes $1 /(4 \delta k)$. Considering the wavenumber-to-wavelength conversion, it becomes (Häusler \& Lindner, 1998)

$$
z_{\max }=\frac{\lambda^{2}}{4 n \delta \lambda}
$$

where $\delta \lambda$ is the wavelength resolution of the spectrometer.

Fourth, the roll-off in depth of the SD-OCT signal is determined by the spectral content (i.e., full-width-at-half-maximum (FWHM) of the transmission response of a single channel) of the spectrometer and the camera pixel size. The imaging range of SD-OCT is limited by the signal roll-off, which is the attenuation of the OCT signal due to washout of the interference fringe visibility with increasing depth. The signal amplitude roll-off function is given by Hu, Pan, and Rollins (2007)

$$
A(z)=\frac{\sin \left(d_{x} \mathcal{R} z\right)}{\left(d_{x} \mathcal{R} z\right)} \exp \left[-\frac{a^{2} \mathcal{R}^{2} z^{2}}{4 \ln 2}\right]
$$

where $d_{x}$ is the pixel width, $R=\delta k / d_{x}$ is the reciprocal linear dispersion of the spectrometer, and $a$ is the spot size at the focal plane of the spectrometer. The sinc and Gaussian functions in Eq. (3) correspond to the Fourier transform (FT) of the square-shaped camera pixels and Gaussian beam profile in the spectrometer, respectively. Considering the sinc function, it is beneficial to have a small pixel size and large linear dispersion (small reciprocal dispersion) in order to get a better signal roll-off. In order to reduce the effect of Gaussian function on signal roll-off, the FWHM diameter of the focused spot size as well as the reciprocal linear dispersion should be reduced which necessitates a grating spectrometer with a high groove density (large number of arrayed waveguides in an AWG spectrometer). By applying wavenumberto-wavelength conversion and after some rearrangements Eq. (3) becomes

$$
A(z)=\frac{\sin \left(\pi z / 2 z_{\max }\right)}{\left(\pi z / 2 z_{\max }\right)} \exp \left[-\frac{\pi^{2} \omega^{2}}{16 \ln 2}\left(\frac{z}{z_{\max }}\right)^{2}\right]
$$

$z_{\max }$ is taken from Eq. (2) and $\left(a / d_{x}\right)$ is defined as $\omega$ in Yun, Tearney, Bouma, Park, and de Boer (2003), which is the ratio of the spectrometer FWHM transmission response of a single channel to the wavelength resolution. 
Finally, for maximum SNR, the spectrometer loss should be minimized in the design stage. Typical SNR values for high-quality OCT imaging are on the order of 100-110 dB (Hammer, 2011).

\section{Passive on-chip OCT components}

\subsection{Optical waveguides}

The basic element of an integrated photonic component is an optical waveguide. Many other components such as splitters, filters, modulators, amplifiers, lasers, and detectors are usually built by introducing modifications to the waveguide structure. The spectrometer and interferometer of an on-chip SD-OCT system consist of multitudes of optical waveguides, which should have good transmission characteristics for the wavelength range associated with the broadband source. They have to be single mode and the overall propagation losses and birefringence have to be low. The index contrast and the waveguide geometry determine the divergence of the free-space beam emerging from sample and reference arms. For the OCT application, the waveguides should be designed to produce a low-divergence output beam while maintaining a small device size. The most commonly used optical waveguide geometry is channel waveguide due to its high fabrication tolerance (see Fig. 1).

Optical waveguides are realized in both low contrast (e.g., silica, polymers) and high contrast (e.g., InP, SOI) material platforms. Low-contrast materials show excellent fiber-to-chip coupling efficiency (i.e., 0.1 dB; Germann et al., 2000) due to the large size of the single-mode waveguides, however they have low integration density due to large bend radius $(\sim 15-20 \mathrm{~mm}$; Bona, Germann, Horst, Offrein, \& Salemink, 1999) required for low loss operation. On the other hand, high contrast systems allow for very small bending radius ( 1-100 $\mu \mathrm{m}$; Lim et al., 1999) with low losses, but they suffer from high fiberto-chip coupling losses (3-10 dB; Cheben et al., 2007) due to small mode field dimensions. Although it may not be possible to realize all of them optimally on the same material system, different components can be combined on a substrate that is suitable to implement passive structures. The on-chip OCT systems that were realized so far have used silicon material platform (including $\mathrm{Si}, \mathrm{SiO}_{2}$, $\mathrm{Si}_{3} \mathrm{~N}_{4}$, and $\mathrm{SiON}$ ) for the passive components (i.e., spectrometer, interferometer and reference arm) while for the light sources and the detectors compound semiconductor materials have been used. Using silica waveguides, Culemann et al. (2000) demonstrated parallel integration of eight Michelson interferometers for time-domain OCT (TD-OCT). Choi et al. (2008) reported an 
A

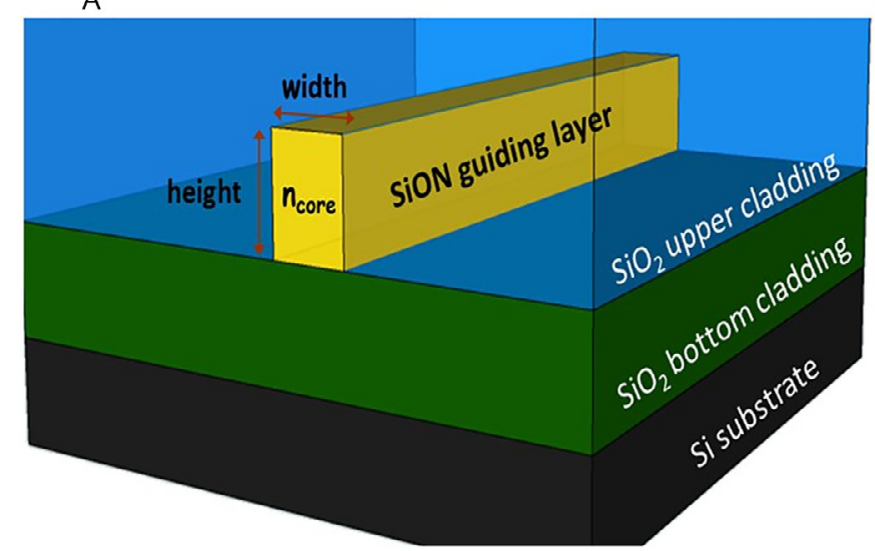

B

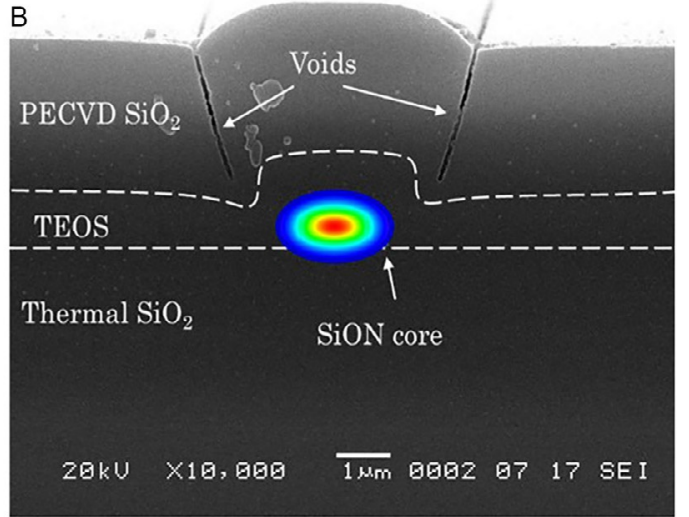

Fig. 1 (A) Schematic diagram of a SiON channel waveguide with flexible design parameters of height, width, and core refractive index ( $\left.n_{\text {core }}\right)$. (B) SEM image of the test structure after top $\mathrm{SiO}_{2}$ cladding deposition with 2D modal field distribution. 
OCT system that uses a 256 channel, $25 \mathrm{GHz}$ channel spacing, silica arrayed waveguide grating (AWG) as a spectrometer. $\mathrm{SiON}$ was a material choice by Akca et al. (2013) to demonstrate a beam splitter and an AWG on the same chip for SD-OCT. Using $\mathrm{Si}_{3} \mathrm{~N}_{4}$ and $\mathrm{SiO}_{2}$ waveguides, Nguyen et al. fabricated a Michelson interferometer for SS-OCT (Nguyen et al., 2012). Use of silicon waveguides for OCT was first demonstrated by Margallo-Balbas et al. (2008) by fabricating a thermo-optic delay line for TD-OCT. Yurtsever et al. realized a silicon integrated Mach-Zehnder interferometer with a long on-chip reference arm (Yurtsever, Považay, et al., 2014).

\subsection{Interferometer}

Interferometer is the common component of all OCT types. Generally, Michelson interferometer (MI) is the preferred interferometer type due to its simple configuration. It is based on a 50:50 (3 dB) optical power splitter. There are several types of integrated optical power splitters that can be used as a beam splitter in an on-chip MI. For high image quality, it is important to design wavelength-insensitive couplers with minimum loss. Directional couplers are the commonly used optical splitters due to their structural simplicity, ease of design, and zero excess loss intrinsically; however, their highly wavelength-dependent operation makes them incompatible for OCT imaging. Many different approaches have been investigated in order to realize wavelength-insensitive $3 \mathrm{~dB}$ couplers (Adar, Henry, Kazarinov, Kistler, \& Weber, 1992; Akca et al., 2012; Chang, 2016; Jinguji, Takato, Sugita, \& Kawachi, 1990; Little \& Murphy, 1997; Louisell, 1954; Lu et al., 2015; Maese-Novo et al., 2013; Wang, Lu, \& He, 2002; Xu et al., 2007). Multi-mode interference (MMI) couplers are broadband optical power splitters, however they suffer from excess loss (Maese-Novo et al., 2013; Xu et al., 2007). Non-uniform adiabatic couplers exhibit very wide bandwidths, however they are rather long (i.e., couple of millimeters) (Adar et al., 1992; Louisell, 1954). Balanced couplers also work over large bandwidths, which are Mach-Zehnder interferometer type structures with high fabrication tolerances (Akca, Doerr, et al., 2012; Jinguji et al., 1990; Little \& Murphy, 1997). Asymmetric directional couplers are compact broadband splitters, which are comprised of waveguides with different waveguide widths or bending radii ( $\mathrm{Lu}$ et al., 2015). Another wavelength-insensitive coupler is a $\mathrm{Y}$ coupler, which is very susceptible to fabrication errors (Wang et al., 2002). Recently, a modified blunt Y coupler design was proposed by Chang et al. to overcome the aforementioned problems of the Y couplers (Chang, 2016). 
In order to test the performance of above mentioned wavelengthinsensitive couplers, we have fabricated non-uniform adiabatic, balanced, MMI, asymmetric directional and modified blunt $\mathrm{Y}$ couplers, using $\mathrm{Si}_{3} \mathrm{~N}_{4}$ rib waveguides. The material system is $200-n m$-thick LPCVD $\mathrm{Si}_{3} \mathrm{~N}_{4}$ film on an $8-\mu \mathrm{m}$-thick thermally-oxidized silicon wafer. A $4.0 \mu \mathrm{m}$ thick $\mathrm{SiO}_{2}$ layer $(n=1.44)$ was deposited by PECVD to complete the waveguiding cross-section. The refractive index of the thermal oxide and $\mathrm{Si}_{3} \mathrm{~N}_{4}$ layer is 1.464 and 2.0 at $1550 \mathrm{~nm}$, respectively. Single-mode rib waveguides with $0.15 \mu \mathrm{m}$ of slab height and $2.0 \mu \mathrm{m}$ of waveguide width were designed. However, during the etching process, rib waveguides were under etched by $\sim 20 \mathrm{~nm}$, which was confirmed by scanning electron microscopy. The propagation loss of the $\mathrm{Si}_{3} \mathrm{~N}_{4}$ waveguides defined by $\mathrm{RIE}$ is around $1.5 \mathrm{~dB} / \mathrm{cm}$ at $1550 \mathrm{~nm}$, which can be reduced by a post-reflow step after etching the $\mathrm{Si}_{3} \mathrm{~N}_{4}$ layer (Nguyen et al., 2011). Characterization of these couplers were performed by coupling TE-polarized light from a supercontinuum light source (NKT SuperK EXTREME, EXR-4) into the input waveguide with a single-mode polarization-maintaining fiber. The output signal was sent to an optical spectrum analyzer (Yokogawa, AQ6370B) through a buttcoupled single-mode fiber. The transmission spectra measured at the output ports of the splitter were normalized with respect to the transmission spectrum of a straight rib waveguide. Each coupler design, simulation and measurement results are given below.

\subsubsection{Non-uniform adiabatic coupler}

A non-uniform adiabatic coupler consists of two tapered waveguides as depicted in Fig. 2A. If the coupler is sufficiently asynchronous at its input, the power in each of the input waveguides will predominantly go into only one of the system modes (i.e., fundamental or the first order). In the coupler discussed here, the synchronicity of the waveguides is arranged in such a way that only the zero-order local system mode of the coupler will be excited. If the widths of the coupled waveguides change adiabatically, i.e., the cross-section of the system changes slowly with the $z$-position, a system mode will propagate through the structure with negligible power conversion to the other system mode. If the widths of the waveguides are interchanged at the end of the tapered coupling region, this gives a full coupler, whereas equal output widths will result in a 3-dB coupler (Adar et al., 1992). The theoretical analysis of adiabatic couplers is given in Louisell (1954). The non-uniform adiabatic coupler has wavelength-flattened performance compared to other couplers, but it is rather long which prevents its widespread use. 

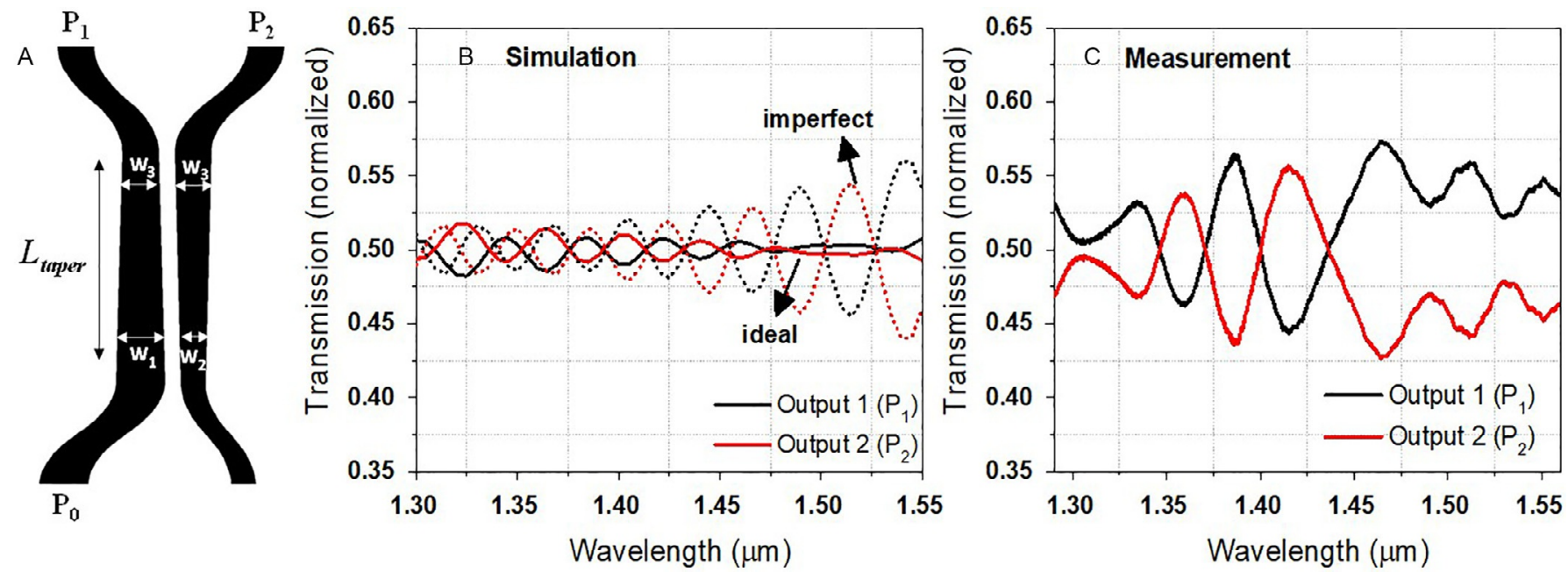

Fig. 2 (A) Schematic of the non-uniform adiabatic coupler with relevant design parameters. (B) Simulated transmission of the non-uniform adiabatic coupler in the ideal case, i.e., without fabrication imperfections, and including under-etched waveguides. (C) Measurement results. 
The aforementioned waveguide geometry was used to fabricate nonuniform adiabatic couplers. The other design parameters are the length of the tapered section $L_{\text {taper }}=8 \mathrm{~mm}$, widths of the input and output waveguides of the tapered sections $w_{1}=1.9 \mu \mathrm{m}, w_{2}=1.3 \mu \mathrm{m}, w_{3}=1.6 \mu \mathrm{m}$, and the separation between waveguides $d=0.9 \mu \mathrm{m}$. An oscillatory transmission response was observed which is attributed to the under etching of the $\mathrm{Si}_{3} \mathrm{~N}_{4}$ rib waveguides. This effect was included in the BPM simulations and the simulation result of the imperfect coupler is shown in Fig. 2B with black and red dotted lines. Similar oscillatory behavior was observed. The $\pm 0.5 \mathrm{~dB}$ bandwidth of $250 \mathrm{~nm}$ was measured as given in Fig. 2C.

\subsubsection{Balanced coupler}

3- $\mathrm{dB}$ balanced couplers can be realized by cascading two conventional directional couplers in a Mach-Zehnder (MZ) configuration with a relative phase shift of $2 \theta$ introduced between them (Little \& Murphy, 1997) (Fig. 3A). In this configuration, the purpose of the second coupler is to compensate for deviations introduced by the first one. The phase shift of $2 \theta$ in the $\mathrm{MZ}$ can be chosen such that the response of the device becomes flatter than a normal 3-dB directional coupler. Cherchi et al. showed geometrically, by means of Poincaré spheres, how the phase shift can effectively compensate small errors in the coupler sections (Cherchi, 2003). Such couplers can be designed to yield a maximally flat response with respect to deviations in wavelength, polarization, or uniform fabrication over a broad spectral range, with no excess loss. The power cross coupling of balanced couplers is given as (Little \& Murphy, 1997):

$$
S=\cos ^{2}(\theta) \sin ^{2}\left(\phi_{1}+\phi_{2}\right)+\sin ^{2}(\theta) \sin ^{2}\left(\phi_{1}-\phi_{2}\right)
$$

A wavelength-flattened design can be obtained by a numerical optimization of $\phi_{1}$, and $\phi_{2}$, and $\theta$. Since $\cos ^{2}(\theta)+\sin ^{2}(\theta)=1$, the cross coupling of a balanced coupler is bounded by two envelope functions, i.e., $\sin ^{2}\left(\phi_{1}+\phi_{2}\right)$ and $\sin ^{2}\left(\phi_{1}-\phi_{2}\right)$ where the weight of these terms depends on the value of $\theta$. Choi et al. (2008) specifies that in order to obtain a 3-dB coupler with a maximally flat wavelength response, the parameters are chosen as $\phi_{1}=\pi / 2$ (full coupler), $\phi_{2}=\pi / 4$ ( $3-\mathrm{dB}$ coupler), and $2 \theta=2 \pi / 3$.

The relevant design parameters are $L_{1}=230 \mu \mathrm{m}, L_{2}=136 \mu \mathrm{m}$, and $\Delta L=0.281 \mu \mathrm{m}$. The BPM simulation result of this coupler is given in Fig. 3B with black and red solid lines. Even though it was designed to work around $1.55 \mu \mathrm{m}$ wavelength range, a significant central wavelength shift, i.e., $\sim 200 \mathrm{~nm}$, was observed. The effect of under etching of the waveguides was 

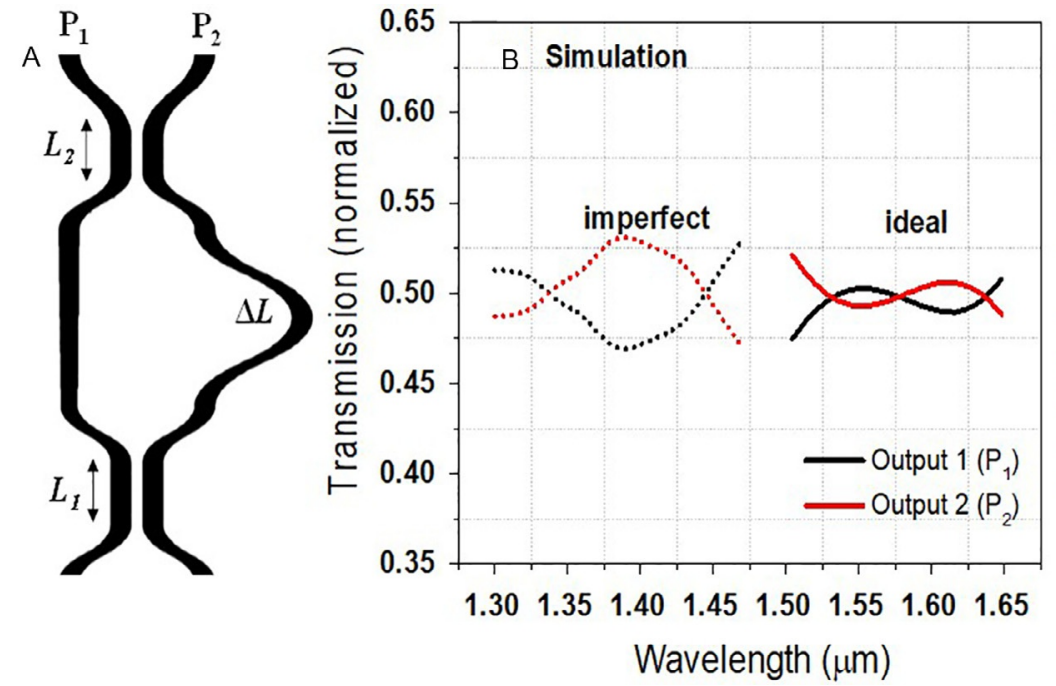

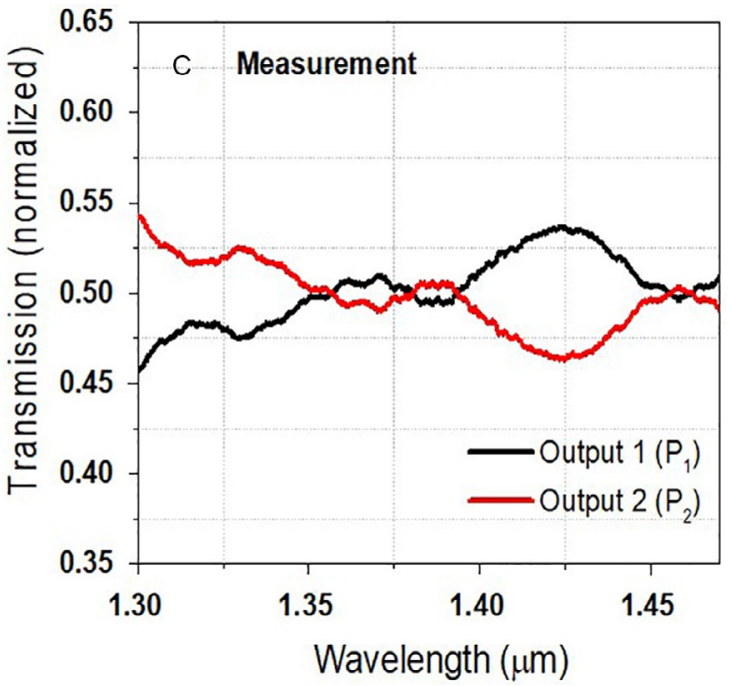

Fig. 3 (A) Schematic of the balanced coupler with relevant design parameters. (B) Simulated transmission of the balanced coupler in the ideal case, i.e., without fabrication imperfections, and imperfect case; i.e., including the effect of under-etched waveguides. (C) Measurement results. 
simulated and the results showed the same wavelength shift (Fig. 3B, labeled "imperfect"). The $\pm 0.5 \mathrm{~dB}$ bandwidth of $170 \mathrm{~nm}$ was measured as given in Fig. 3C.

\subsubsection{Asymmetric directional coupler design}

The asymmetric directional coupler proposed here consists of $\mathrm{S}$ bends, tapered waveguides and straight waveguides with different waveguide widths as given in Fig. 4A. In the proposed coupler design, two coherent light beams with a certain amplitude ratio is produced in the end of the first tapered waveguide region. $L$ is used to adjust the phase difference between the two coherent beams. The output power-coupling ratio is then controlled by the second tapered waveguide section. In this approach, the lengths of the tapered regions and the phase section are optimized in order to cancel the dispersion and thereby make this design wavelength insensitive.

3D BPM was used to design the asymmetric directional coupler. First, the separation between waveguides was chosen to be $d=1 \mu \mathrm{m}$ which is within the fabrication limits. The widths of the tapered waveguides were set to be $w_{a}=2.1 \mu \mathrm{m}, w_{b}=1.5 \mu \mathrm{m}$ for inducing enough phase difference between coupler arms while assuring single-mode operation of the waveguides. The length of the tapered section was defined to be $L_{\text {taper }}=30 \mu \mathrm{m}$ for the adiabatic transition. The length of the phase section was swept from 0 to $100 \mu \mathrm{m}$ in the BPM simulations, and for each length value, a wavelength scan was made in order to compare the flatness of the splitting ratio at each phase section length. Finally, the optimal length of the phase section is obtained as $L_{p s}=48 \mu \mathrm{m}$. Fig. $4 \mathrm{~B}$ shows the BPM simulation results of the ideal and imperfect asymmetric directional couplers with solid and dotted lines, respectively. The $\pm 0.5 \mathrm{~dB}$ bandwidth range was obtained as $90 \mathrm{~nm}$ as given in Fig. 4C.

\subsubsection{Multi-mode interference (MMI) coupler}

The working principle of a MMI coupler is based on self-imaging principle. The main structure of the MMI coupler (Fig. 5A) is a multimode waveguide, which supports several modes with different mode propagation constants $\beta_{m}=2 \pi n_{\text {eff, } m} / \lambda$ where $n_{\text {eff, } m}$ is the effective refractive index of the $m$ th mode. The beat length $\left(L_{\pi}\right)$ between the two lowest order modes, i.e., $L_{\pi}=\pi /\left(\beta_{0}-\beta_{1}\right)$, can be expressed in terms of its wavelength dependence (Maese-Novo et al., 2013)

$$
L_{\pi}(\lambda)=\frac{4 n_{f} W_{M M I}^{2}}{3 \lambda}
$$



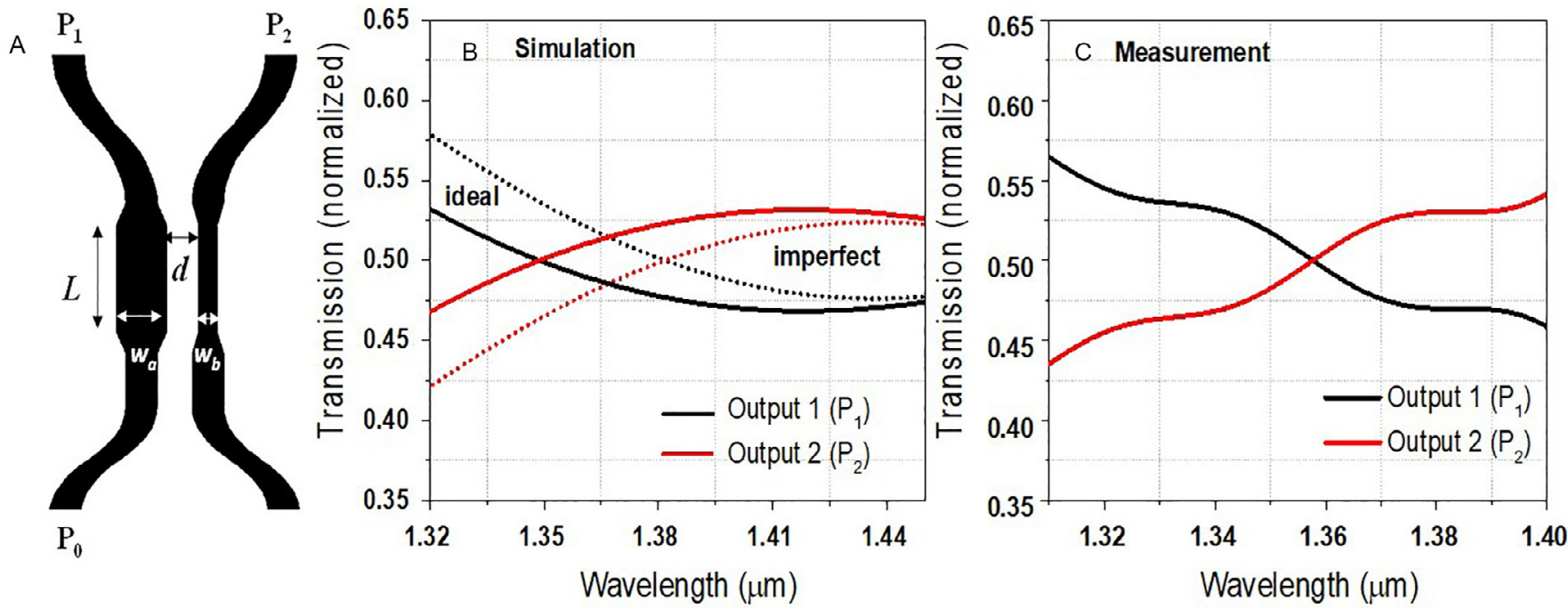

Fig. 4 (A) Schematic of the asymmetric directional coupler with relevant design parameters. (B) Simulated transmission of the ideal case, i.e., without fabrication imperfections, and imperfect case, i.e., including the effect of under-etched waveguides. (C) Measurement results of the fabricated device. 

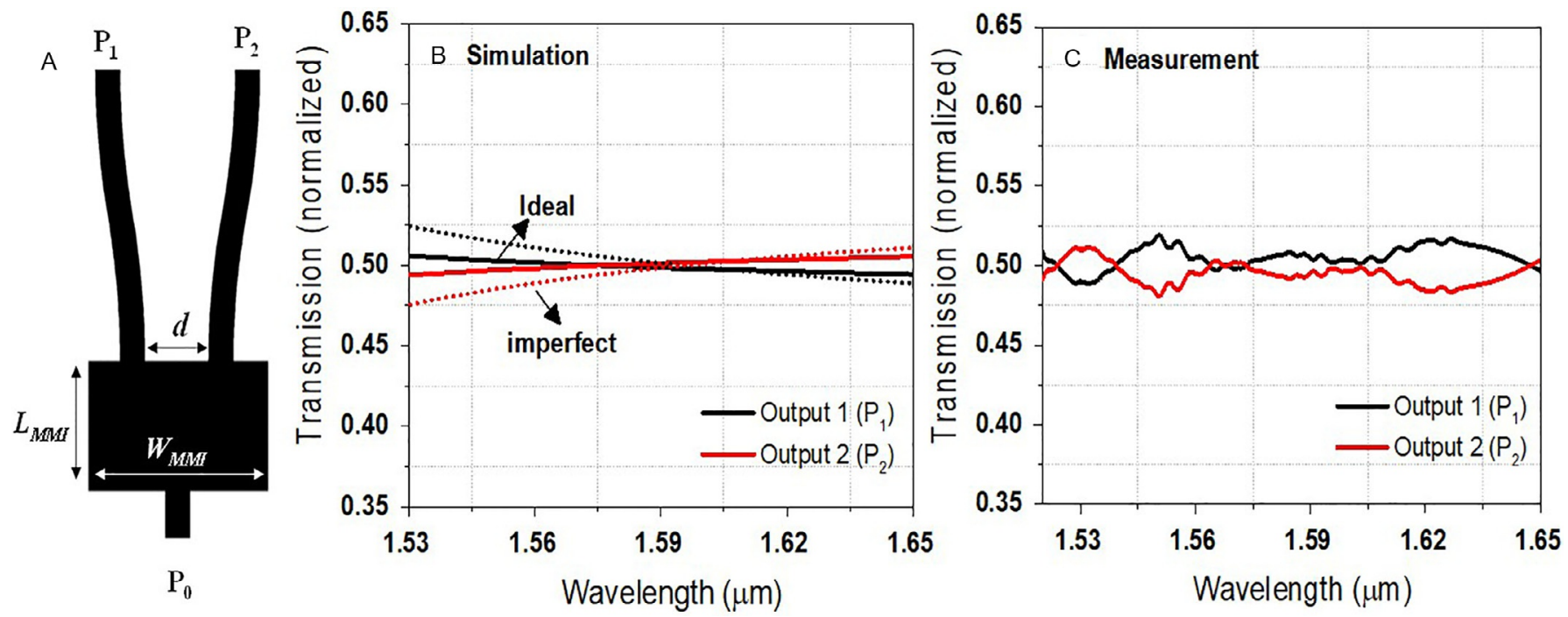

Fig. 5 (A) Schematic of the MMI coupler with relevant design parameters. (B) Simulated transmission of the MMI coupler in the ideal case, i.e., without fabrication imperfections, and imperfect case; i.e., including the effect of under-etched waveguides. (C) Measurement results. 
where $n_{f}$ is the equivalent index of the multimode region, and $W_{M M I}$ is the width of the multimode region. Wavelength dependence of the beat length is the main source of the MMI bandwidth limitation. The width and the length of the multimode region of the fabricated MMI were calculated to be $W_{M M I}=20 \mu \mathrm{m}, L_{M M I}=236 \mu \mathrm{m}$. The output waveguide separation was $d=1 \mu \mathrm{m}$.

The BPM simulations of the ideal (solid lines) and the imperfect case (dotted lines) due to fabrication problems are given in Fig. 5B. In the measurement results an oscillatory behavior was observed which cannot be explained by the under-etched waveguide geometry (Fig. 5C). One possible reason could be the back reflection of the light at the multimode and single-mode waveguide interfaces, which cannot be simulated using BPM. The $\pm 0.5 \mathrm{~dB}$ bandwidth of $130 \mathrm{~nm}$ and excess loss value of $2 \mathrm{~dB}$ were measured.

\subsubsection{Modified blunt $Y$ coupler}

Conventional Y couplers have very large working wavelength range; however, their operation is very sensitive to the fabrication errors. In order to achieve a lossless broadband Y coupler, an infinitely sharp vertex between output waveguides is needed. However, such a sharp feature cannot be fabricated due to the finite fabrication resolution. As a solution to fabrication sensitive operation of conventional Y couplers, blunt Y couplers were proposed which suffer from increased loss (Chang, 2016). Chang et al. proposed a modified blunt $\mathrm{Y}$ coupler with a wider output slab (Fig. 6A), which exhibits lower loss and lower susceptibility to fabrication variations.

The modified blunt Y coupler designed in this work, has the input waveguide width of $W_{\text {in }}=2 \mu \mathrm{m}$, and gap between the output waveguide of $g=0.8 \mu \mathrm{m}$. By scanning the output width of the tapered region, i.e., $W_{\text {out }}$, at two wavelengths, i.e., 1400 and $1600 \mathrm{~nm}$, the optimum value was obtained as $W_{\text {out }}=6.75 \pm 0.25 \mu \mathrm{m}$ (see Fig. 6B). The length of the adiabatic region is $L_{\text {taper }}=200 \mu \mathrm{m}$ which ensures the lossless transition between input and output waveguide modes. The simulation results of this coupler are given in Fig. 6C with solid lines, where dotted lines show the imperfect design due to fabrication problems. A similar oscillatory response that was seen in the MMI coupler was observed which is possibly due to the same back reflection problem. The $\pm 0.5 \mathrm{~dB}$ bandwidth range of $300 \mathrm{~nm}$ and excess loss value of $0.5 \mathrm{~dB}$ were obtained as given in Fig. $6 \mathrm{D}$.

In summary, five different broadband coupler configurations have been designed and realized in $\mathrm{Si}_{3} \mathrm{~N}_{4}$ platform. The effect of under-etched 

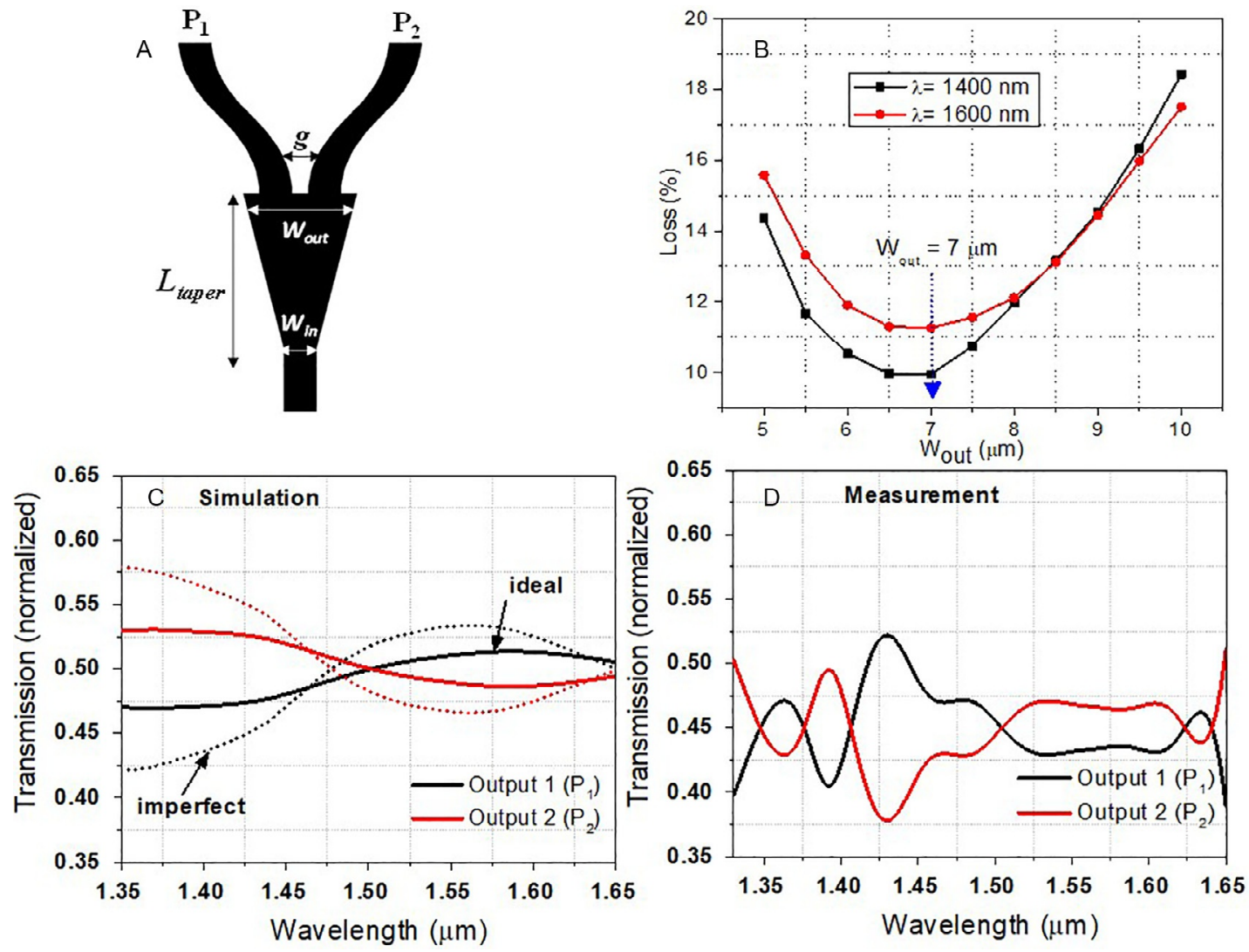

Fig. 6 (A) Schematic of the modified blunt $Y$ coupler with relevant design parameters. (B) Calculated loss values for 1400 and $1600 \mathrm{~nm}$ wavelengths. The device has the minimum loss when $W_{\text {out }}=6.75 \pm 0.25 \mu \mathrm{m}$ for both wavelengths. (C) Simulated transmission of the coupler in the ideal case, i.e., without fabrication imperfections, and imperfect case; i.e., including the effect of under-etched waveguides. (D) Measurement results. 
$\mathrm{Si}_{3} \mathrm{~N}_{4}$ rib waveguides was included in the BPM simulations and compared with the experimental results. The non-uniform adiabatic coupler showed a very large bandwidth (i.e., $250 \mathrm{~nm}$ ) with no excess loss; however, the device length was rather long (i.e., $8 \mathrm{~mm}$ ). The MMI coupler suffered from high excess loss (i.e., $2 \mathrm{~dB}$ ) and internal back reflections despite its large bandwidth (i.e., $130 \mathrm{~nm}$ ) and compact footprint (i.e., $350 \mu \mathrm{m}$ ). The modified blunt $\mathrm{Y}$ coupler showed the largest bandwidth range (i.e., $320 \mathrm{~nm}$ ) and small excess loss (i.e., $0.5 \mathrm{~dB}$ ), however it is a $1 \times 2$ coupler similar to abovementioned MMI coupler; therefore, it can be used only in certain circumstances. The asymmetric directional coupler had the smallest footprint (i.e., $200 \mu \mathrm{m}$ ); however its operation bandwidth was also the smallest (i.e., $90 \mathrm{~nm}$ ). Among all designs, the balanced coupler exhibited the best performance in terms of bandwidth (i.e., $170 \mathrm{~nm}$ ), and excess loss (i.e., $0 \mathrm{~dB}$ ) at a moderate device length $(1.8 \mathrm{~mm})$.

\subsection{Spectrometer}

The spectrometer is the principal component of SD-OCT systems. Each of its parameters can have a dramatic effect on overall system performance. The axial resolution, maximum imaging range, and signal roll-off of an SD-OCT system are all dependent on the spectrometer's design. It is an expensive component, which usually consumes a lot of space. Additionally it is fragile and susceptible to miscalibration. Therefore, this part has to be integrated on a chip. The complexity of the spectrometer and its desired resolution and efficiency make this component the most challenging one to fabricate on a chip. Monolithic spectrometers have been made, although not at the required spectral resolution (Avrutsky, Chaganti, Salakhutdinov, \& Auner, 2006; Goldman, White, \& Anheier, 1990; Sander, Blume, \& Müller, 1996; Sander \& Mueller, 2001). Echelle grating spectrometers can provide high resolution; however, there are several issues, which limit the common use of echelle gratings such as facet-roughness related losses, polarization dependence of the grating efficiency, and birefringence (Cheben, 2007).

Although integration of a spectrometer on a chip is challenging, arrayed waveguide gratings (AWGs) present a well-established way toward miniaturization. Besides their extensive usage in telecommunication for (de)multiplexing (Smit \& van Dam, 1996), AWGs are also ideally suited for applications such as OCT and spectroscopy, with their high spectral resolution, small form factor, large bandwidth, and low insertion loss. In addition to their advantages listed above, AWGs are cost-effective, which makes 
them favorable for integration with SD-OCT systems. However, there are design limitations on resolution and free spectral range (FSR), which restrict the axial resolution and maximum imaging range of SD-OCT systems. By applying different approaches (e.g., cascading or interleaving several AWGs), these limitations can be overcome (Akca \& Doerr, 2019; Nikbahkt, Karabiyik, \& Akca, 2020; Takada, Abe, Shibata, \& Okamoto, 2001; Takada, Abe, Shibata, \& Okamoto, 2002a, 2002b; van Wijk, Doerr, Ali, Karabiyik, \& Akca, 2020).

An AWG consists of input/output waveguides, two focusing slab waveguides and a phased array of multiple waveguides with a constant path length difference $\Delta L$ between neighboring waveguides as shown in Fig. 2A. The operation of an AWG (Smit \& van Dam, 1996) is briefly explained, referring to Fig. 7A. Light from an input waveguide becomes laterally divergent in a first free propagation region (FPR) and illuminates the input facets of an array of waveguides with a linearly increasing length that leads to their typical curved shape. For a central design wavelength $\lambda_{c}$ the phase difference at the output facets of adjacent array waveguides is an integer multiple of $2 \pi$. Since these facets are arranged on a circle, a cylindrical wavefront is formed

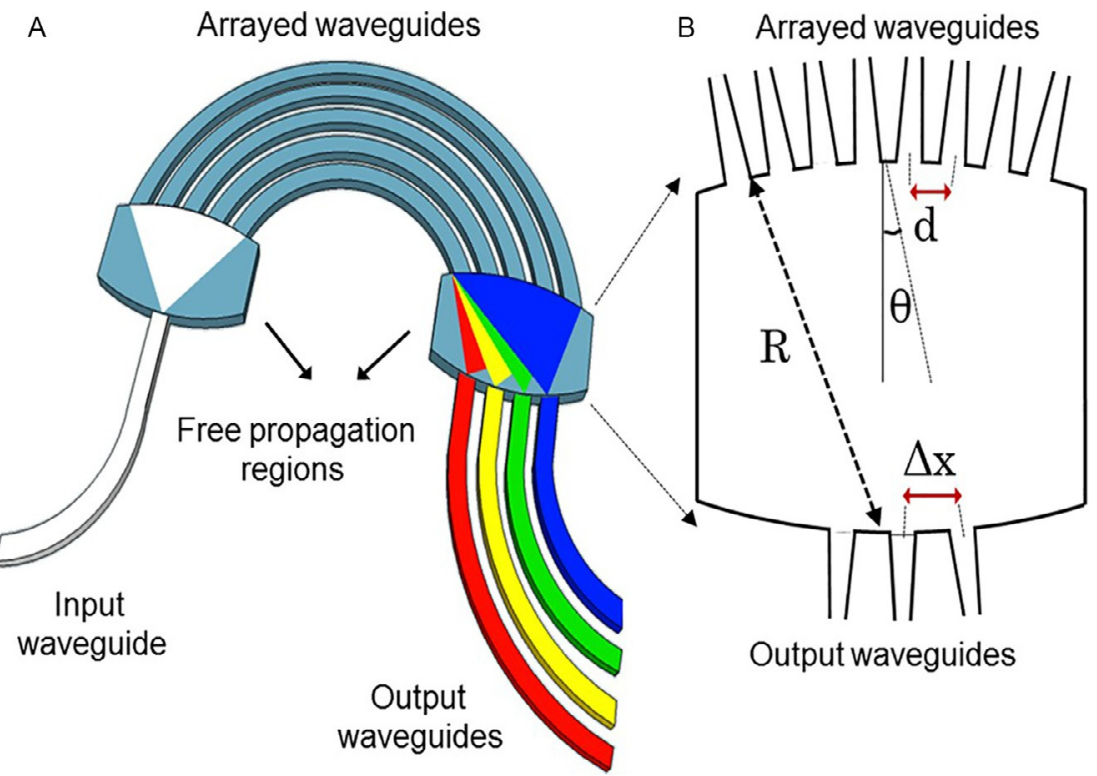

Fig. 7 (A) Schematic layout of an arrayed waveguide grating (AWG). (B) Geometry of the receiver side of the AWG (second FPR) and definition of parameters $\Delta x, d, R, \theta$ : see Sander et al. (1996). 
at the beginning of a second FPR, which generates a focal spot at the central output waveguide. The phase shift caused by the length differences between arrayed waveguides is linearly dependent on wavelength, the resulting wavelength-dependent phase gradient implies a tilt of the cylindrical wavefront at the beginning of the second FPR, which causes the focal spot to shift to a different output waveguide with wavelength.

\subsubsection{AWG parameters for OCT imaging}

The axial resolution of an SD-OCT system using an AWG is determined by the effective bandwidth of the detected spectrum, which depends on both the bandwidth of the light source and the FSR of the spectrometer. Matching the bandwidth of the AWG transmission function with the bandwidth of the light source is the most economical configuration, since for a given source bandwidth the axial resolution will not improve if the bandwidth of the AWG transmission function is made much larger than this bandwidth, and vice versa. The maximum useful transmission bandwidth of an AWG is its FSR, which is valid for loss nonuniformity $\leq 3 \mathrm{~dB}$. In practice, the FSR of an AWG is limited by the size of the device (Smit \& van Dam, 1996). By considering the design limitations, the optimum FSR values can be chosen.

The choice for a maximum depth range is a compromise between various imaging performance parameters. Given a fixed size of the AWG imaging plane, a larger imaging depth can be obtained by using an AWG with higher resolution; however, this would result in a lower optical bandwidth and, hence lower spatial OCT resolution. Smaller spacing between adjacent waveguides results in an increased crosstalk and, consequently broader beam sizes on the CCD and thereby more signal roll-off in depth.

For an SD-OCT system with an AWG spectrometer, the roll-off in depth is determined by the relative spectral content (i.e., FWHM of the transmission response of a single channel) of the AWG output channels. As the AWG spatially disperses the input light over the output plane of the second FPR, the spectral content is limited by the size of the output waveguides. However, this spectral content in a single output waveguide can be increased due to diffraction-limited focusing of the light onto the output channel, crosstalk between output waveguides, and fabrication imperfections, which results in a degradation in signal roll-off. For an AWG-based SD-OCT system, light is sampled twice; first at the focal plane of the AWG by the discretely located output waveguides and second by the camera pixels. The first sampling due to AWG output channels adds an extra sinc factor, 
which is the Fourier transform of the rectangular-shaped output waveguides. The modified formula of sensitivity roll-off in case of an AWG spectrometer is given in Eq. (4). In order to avoid severe signal roll-off in depth, $\omega$ is chosen smaller than 1 , which necessitates an adjacent-channel crosstalk value of less than $10 \mathrm{~dB}$. The desired crosstalk value is achieved by setting the spacing between the output waveguides in the focal plane of the AWG spectrometers accordingly.

For maximum SNR, the AWG spectrometer loss is minimized by applying linear tapers at the interfaces of arrayed and input/output waveguides with the FPRs. Ideally, the gaps near the FPR between arrayed waveguides should approach zero in order to capture more light and, thereby, reduce the excess loss. This would, however, result in extremely sharp features that cannot be accurately reproduced by lithographic processes; therefore, the gap width was chosen to be $1 \mu \mathrm{m}$ for the arrayed waveguides for all AWG spectrometers. The taper width of the input/output waveguides was determined as a compromise between loss and adjacent-channel crosstalk; the larger the taper width, the lower the excess loss and the higher the crosstalk.

As crosstalk arises from evanescent coupling between output waveguides, it decreases with increasing spacing. However, this leads to increased device size and, therefore, needs to be carefully designed. Acceptable minimum spacing between the arrayed waveguides and between the output waveguides were found by simulating device behavior using the 2-D BPM in order to have an excess loss value of $\leq 3 \mathrm{~dB}$ (for the central channels) and a crosstalk value of $\leq-20 \mathrm{~dB}$.

\subsection{Reference arm}

Reference arm is necessary for acquiring signals with high SNR value. The length of reference arm can be easily changed in a fiber-based OCT setup. The on-chip counterpart of this component also has to be dynamically tunable. In a recent work, a SS-OCT system with an on-chip reference arm was demonstrated (Bourquin et al., 2001). In this work, due to the relatively short length of the reference arm, the sample needed to be scanned to obtain cross-sectional images. Yurtsever et al. solved this problem by using a long on-chip reference arm fabricated first in $\mathrm{Si}_{3} \mathrm{~N}_{4}$ (Yurtsever, Považay, et al., 2014) and later in silicon (Yurtsever, Weiss, et al., 2014). Having a static reference arm on-chip has two major drawbacks; first, dispersion compensation becomes more complicated, second, the reference arm power cannot be optimized to achieve the highest SNR in contrary to a fiber-based system. 
One major advantage of integrated optics is that the operation of the existing optical components can be reconfigured by controlling the material properties using temperature, voltage, or pressure.

As a solution to above issues, we designed power- and length-adjustable on-chip reference arms. Several wavelength-independent full couplers are placed on the reference light pathway, which are used as temperaturecontrolled switches. By using these couplers, the propagating light will be steered toward a new path that has a different optical length and thereby the length of the reference arm will become adjustable. By changing the applied temperature on the heater, the power on the reference arm can be adjusted to any desired value needed for the highest SNR. The example considered here is designed in silicon oxynitride ( $\mathrm{SiON}$ ) material platform as it is being a well-proven system for on-chip OCT applications; however, it can be applied to any material technology as long as it has a reasonably high thermo-optic coefficient.

\subsubsection{Micro-chip layout}

An illustration of the adjustable on-chip reference arm for OCT applications is shown in Fig. 8. The dashed outline indicates the margins of the integrated chip. Here a central wavelength of $1300 \mathrm{~nm}$ and a wavelength range of $100 \mathrm{~nm}$ are aimed at. Input light will be divided into two arms with an integrated $3 \mathrm{~dB}$ adiabatic coupler; half toward the sample arm, half toward the reference arm which is comprised of several multi-S-shaped (radius $R$ ) and straight (length $L_{\mathrm{s}}$ ) waveguides. The back reflected light from the sample will be collected by another waveguide which is placed adjacent to the delivering one. In this way, the amount of available light coming from the sample will be increased significantly. For this specific design, five wavelengthindependent couplers were placed on the reference arm which can be increased to any desired number. The coupling ratio of the coupler can be tuned from $0 \%$ to $100 \%$ by using thermo-optic effect, which is a very useful feature for achieving high SNR. The initial length of the reference arm was chosen to be $20 \mathrm{~cm}$ in this design, which will be extended by $8 \mathrm{~cm}$ (for $L_{\mathrm{s}}=1 \mathrm{~cm}$ ) by steering the reference light toward different pathways with different optical lengths using the switching mechanism. By using more switches, the overall length of the reference arm can be increased further. Moreover, by decreasing $L_{\mathrm{s}}$, the reference arm length can be finely tuned. The light coming from the open end of the reference arm will be used for dispersion control. The straight sections on the multi-S-shaped path (i.e., $L_{\mathrm{s}}$ ) make the whole design flexible by providing the required adjustable optical length while the 


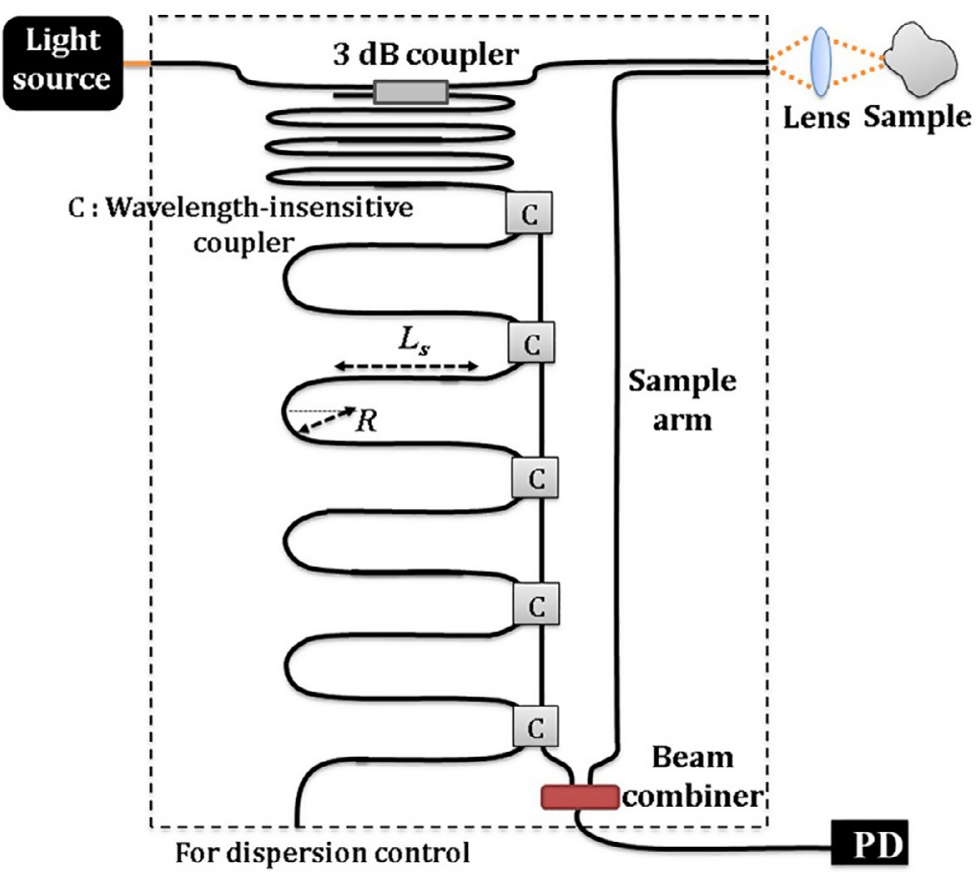

Fig. 8 Schematic of the adjustable on-chip reference arm design. The input light is divided by an adiabatic $3 \mathrm{~dB}$ coupler and sent toward two different paths; the reference arm with several S-shaped (radius $R$ ) and straight waveguides $\left(L_{\mathrm{s}}\right)$ and the sample arm with a straight waveguide. There are five temperature-controlled wavelength-insensitive couplers $(C)$ on the reference arm that act as optical switches. They keep the light on the same arm when no temperature is applied whereas cross-couple the light when there is a $\pi$ phase difference due to thermo-optic effect. The reference and sample arm lights will be combined at the beam combiner and sent to a photodetector (PD).

curved waveguides ensure a compact device size. The light coming from the reference and the sample arms will interfere at the on-chip beam combiner and sent to a matched photodetector.

The proposed adjustable on-chip reference arm design was simulated for $\mathrm{SiON}$ material platform as it is being one of the well-proven material technologies for on-chip OCT applications in addition to its tunable refractive index and large wavelength transparency range (Wörhoff, Klein, Hussein, \& Driessen, 2008). The material system is 1 - $\mu$ m-thick SiON layer deposited on top of an 8- $\mu$ m-thick thermal oxide layer using plasma-enhanced chemical vapor deposition (PECVD) technique. The refractive index of the $\mathrm{SiON}$ layer is 1.535 at $1300 \mathrm{~nm}$, and its thermo-optic (TO) coefficient is $2.35 \times 10^{-5} / \mathrm{K}$ (Chuang \& Liao, 2009). Single-mode channel waveguides 
with a $2.2-\mu \mathrm{m}$ width, $1-\mu \mathrm{m}$ height, 1.535 core refractive index, and 1.4485 cladding refractive index, enabling bending radii down to $0.5 \mathrm{~mm}$, were designed. The effective refractive index of the waveguide stack was calculated to be 1.472 for TE polarization. A $500-$ nm-thick $\mathrm{SiO}_{2}$ top cladding will be used to prevent propagation losses induced by the electrical heaters. BPM simulations were performed for designing and optimizing the optical components.

\subsubsection{Wavelength-insensitive coupler design}

The wavelength-independent couplers used in this design are MachZehnder-type interferometric couplers as explained in Interferometers section. The lengths of the straight coupler sections were calculated as $L=155 \mu \mathrm{m}$, and the delay length was found to be $\Delta L=0.29 \mu \mathrm{m}$. The overall length of the wavelength-insensitive coupler is $1.6 \mathrm{~mm}$. The simulated splitting ratio between the two arms stays constant over $100 \mathrm{~nm}$ bandwidth for both couplers. The coupling ratio remains the same even after a certain temperature applied on the straight section of the coupler (Fig. 9D, bottom).

Wavelength-independent couplers used on the reference arm were designed to act as temperature-controlled switches. A heater was placed on top of the straight section of the coupler. With applied temperature, the effective refractive index of the straight waveguide section is locally increased due to the thermo-optic effect, which induces a phase difference between coupler arms. When the heater is off, the light on both coupler arms will be in phase and the input light will stay in the same arm (Fig. 9C left side). At a certain temperature value (i.e., $T=102^{\circ} \mathrm{C}$ for this design), a $\pi$ phase difference will be generated between coupler arms which steers the input light to the other arm (Fig. 9C right side). It is also possible to achieve switching operation using electro-optic effect or pressure-induced refractive index change by choosing the material technology accordingly.

The design of the wavelength-independent coupler was made in two steps. First, lengths of directional couplers and the delay part were calculated. Second, the coupler designed in the first step was used to simulate the required refractive index increment for a $\pi$ phase difference by scanning the refractive index difference $(\Delta n)$ between coupler arms from 0 to $5 \times 10^{-3}$ with $10^{-4}$ step size. As depicted in Fig. $9 \mathrm{~B}$, for $\Delta n=2.4 \times 10^{-3}, 96 \%$ of the input light cross-coupled to the other arm. The required temperature to induce this refractive index difference was calculated to be $T=102^{\circ} \mathrm{C}$. For five different reference lengths as considered in this design, the on-chip OCT system size 
A

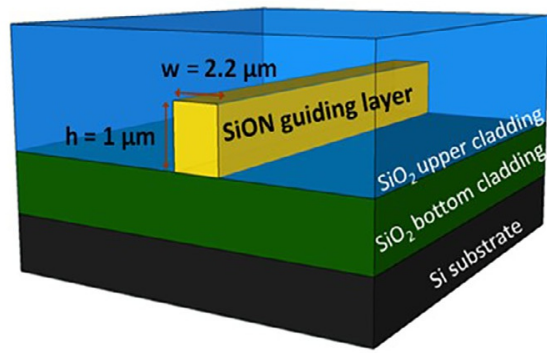

C

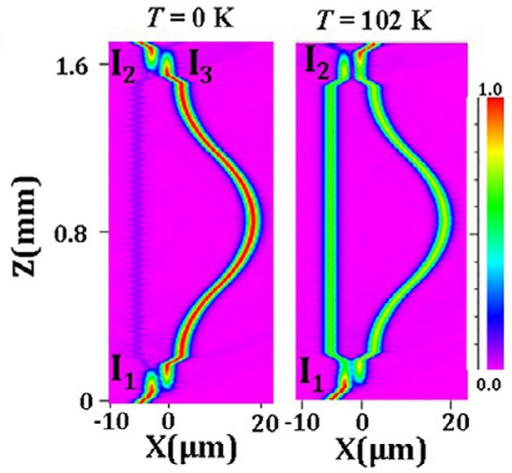

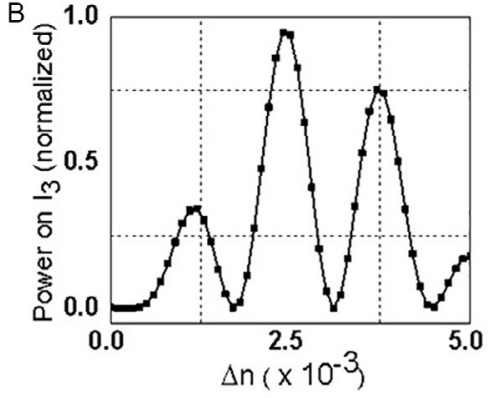

$\mathrm{D}$
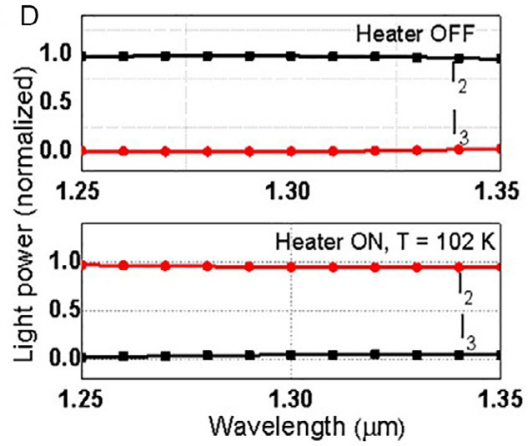

Fig. 9 (A) The schematic of the channel waveguide geometry. (B) Refractive index difference versus power on the cross-coupled coupler arm $\left(I_{3}\right)$. For $\Delta n=2.4 \times 10^{-3}, 96 \%$ of the input light is cross-coupled to the other coupler arm. (C, right) Heater is off, the input light will stay in the same arm. (C, left) Heater is on, $T=102 \mathrm{~K}$, a $\pi$ phase difference will be generated between coupler arms and the input light will cross-couple to the other arm. (D) The coupler is wavelength independent for a wavelength range of $100 \mathrm{~nm}$, and its wavelength independency does not change after the heater is turned on.

was calculated to be around $2 \mathrm{~cm} \times 2 \mathrm{~cm}$. By using a high-index-contrast material system (e.g., silicon or silicon nitride), the device size can be reduced significantly.

\subsection{Sample arm}

The sample arm contains the transverse scanning mechanism and focusing optics. It is responsible for transmitting and receiving light between the sample and the system. Therefore, it is important to choose components that will provide the necessary scanning range, transverse resolution, and scan speed. Integrated optics opens the possibility of considerably reducing the scanning requirements in one direction by using multiple output waveguides located close to each, thus providing parallel signal acquisition. 
In a parallel-OCT system, more the number of the sample arms higher the imaging speed and larger the field of view. However, as the number of sample arms increases, the optical power at each location will get smaller, which will limit the overall system SNR and degrade the imaging performance. One solution to this problem is using a high power light source, which is either too expensive or not commercially available at all. As an alternative and affordable solution, we proposed to use a sequentially switched parallel-OCT approach. Here, instead of having all sample arms available simultaneously (which reduces the output power at each sample arm), a certain number of them, i.e., bundle, will be used at each time. By using an optical switch between each bundle, all sample arms will be sequentially scanned eventually (Fig. 10A). The optical switch can be based on thermal or electro-optic effect. The latter option would not degrade the imaging speed, as the switching speed will be in the GHz range. With this method, the SNR of the OCT system will not degrade either. To do so, a thermal optic switch, which works over a broad wavelength range (i.e., 1.25-1.4 $\mu \mathrm{m}$ ) was designed. The design and simulation results are given in Fig. $10 \mathrm{~B}$ and C. Two adiabatically tapered $3 \mathrm{~dB}$

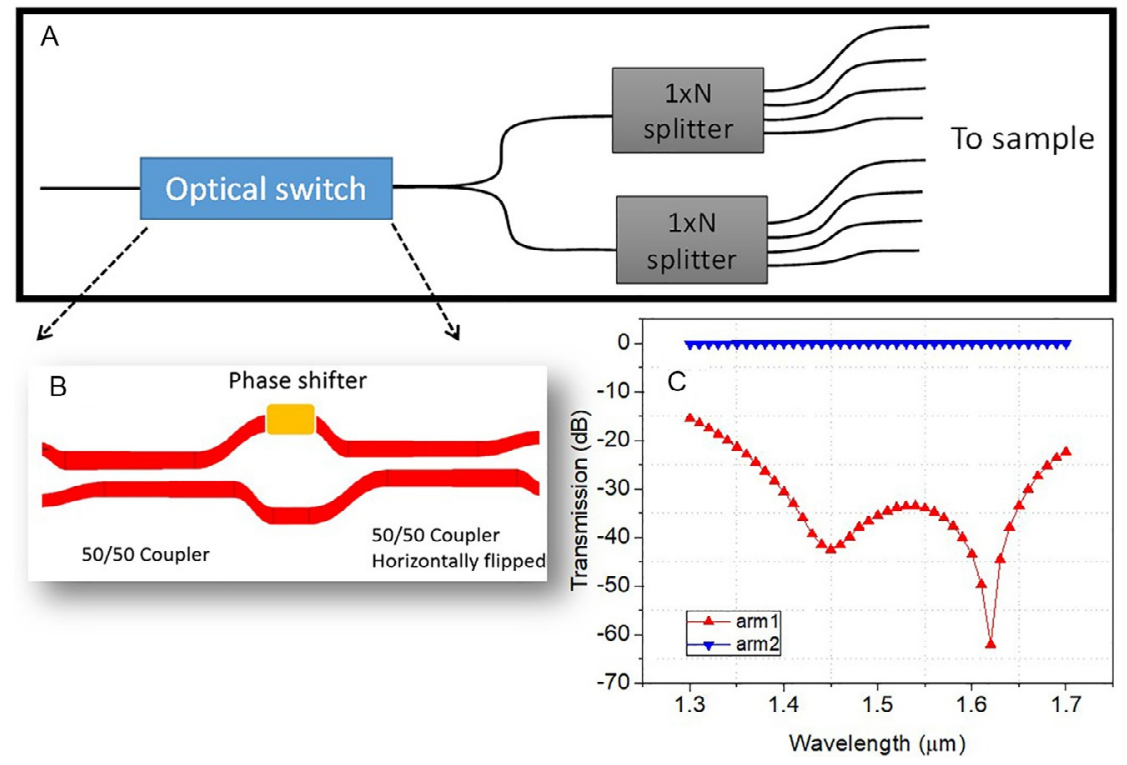

Fig. 10 (A) Sequentially switched parallel-OCT design. Only two stages are shown for ease of understanding. The optical switch will steer the beam from one sample arm bundle to the next by applying voltage to the heater that is placed at the straight section of the optical switch. (B) Optical switch based on thermal effect. (C) Transmission response of the optical switch. 
couplers are combined together using circular waveguides. Two electrodes were placed on the adiabatic sections of the couplers, where the necessary phase shift $(\pi)$ for the switching is created through resistive heating and accompanying local effective refractive index increase. The overall switch length is only $0.8 \mathrm{~mm}$, and for a temperature increase of $100^{\circ} \mathrm{C}$, all power will be transferred from one port to the other. Only one switch and two bundles were used to prove the working principle of the idea as shown in Fig. 10A. By using more switches and bundles, it is possible to obtain a much larger field of view.

\section{OCT imaging with a partially-integrated SD-OCT system}

By combining an AWG spectrometer with an adiabatic coupler, we demonstrated the first partially-integrated SD-OCT system (Akca et al., 2013). An AWG operating at a center wavelength of $\lambda_{c}^{A W G}=1250 \mathrm{~nm}$, with a large FSR of $136 \mathrm{~nm}$ and a high overall wavelength resolution of $\delta \lambda=0.21 \mathrm{~nm}$ was designed by choosing a grating order $m=9$ and $\Delta L=7.6 \mu \mathrm{m}$. The output waveguides of the AWG were removed to increase the spectral resolution and hence the imaging depth range from 0.5 to $2 \mathrm{~mm}$ in air. An adiabatic coupler with a weak wavelength dependence over a range larger than $150 \mathrm{~nm}$ was designed. The coupler and AWG were fabricated in SiON. The integrated chip was interfaced to the light source, external reference arm, two-dimensional scanning system, and linescan camera (Fig. 11) and the system performance was analyzed. An in-tissue depth range of $1.4 \mathrm{~mm}$ and axial resolution of $7.5 \mu \mathrm{m}$ was achieved. Close to the zero delay where both interferometer arms are equal in length, an SNR of $74 \mathrm{~dB}$ was measured for $0.5 \mathrm{~mW}$ of optical power on the sample.

A schematic of the SD-OCT system is shown in Fig. 11. Partially polarized light from a broadband superluminescent diode (Previum, Thorlabs) with a 1320-nm center wavelength, full-width-at-half-maximum bandwidth of $100 \mathrm{~nm}$, and a fiber-coupled optical power of $5.5 \mathrm{~mW}$ was launched via a butt-coupled fiber into one arm of the directional coupler, delivering approximately $0.5 \mathrm{~mW}$ to both, the reference and sample arm. Light polarization was adjusted to maximize the interference signal at the detector. Light exiting the reference and sample arms was collimated by $\times 40$ objective lenses with a numerical aperture (NA) of 0.65 and directed toward reference mirror and sample, respectively. Returning light was focused by the respective objective lenses into the waveguides and passed through the coupler to the AWG. Its output was imaged by a $\times 20$ objective lens $(\mathrm{NA}=0.40)$ onto the entire, 


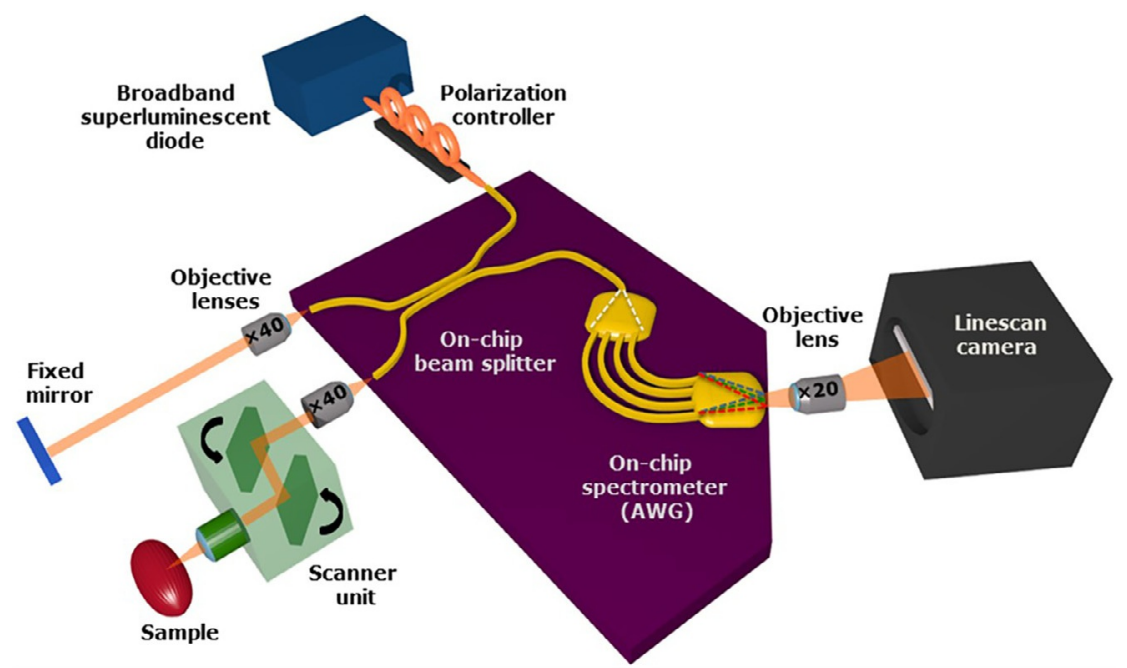

Fig. 11 Schematic of the on-chip SD-OCT system with an external mirror in the reference arm. The output channels of the AWG have been removed in order to have a continuous spectrum. A 3-dB non-uniform adiabatic coupler is used to split the incoming light equally toward sample and reference arms. The light reflected off the sample and the reference arms merge in the coupler and enter the input waveguide of the arrayed waveguide grating (AWG). The dispersed light is imaged onto the entire linescan camera using a $\times 20$ objective lens. Note that the purple region is artificially magnified for viewing purposes.

2.5-mm-wide array of an infrared linescan camera (SUI1024-LDH-1.7RT0500/LC, 1024 pixels, $25 \times 500 \mu^{2}$ pixel size, Goodrich) with a magnification factor of $\sim 10: 1$. The lateral resolution was limited by the NA of the scan lens and the incident beam size to $20 \mu \mathrm{m}$.

The feasibility of on-chip OCT was demonstrated by in vivo imaging of scar tissue. Fig. 12 shows the en face and the corresponding cross-sectional tomogram taken from the scar tissue at the index finger of a volunteer. Cross-section displaying the irregularities caused due to an incision after complete healing is given in Fig. 12A. In the en face view of the region at the layer of the stratum corneum the typical matrix-like distribution of sweat ducts (bright spots) appears on the left and right portion of Fig. 12B. The tissue distortions become obvious at the layer of the living epidermis and the dermal papillae as shown in Fig. 12C. The fine dark lines on the right parallel to the scar correspond to deep folds in the epidermis that also cast shadows onto the deeper dermis (Fig. 12D). 

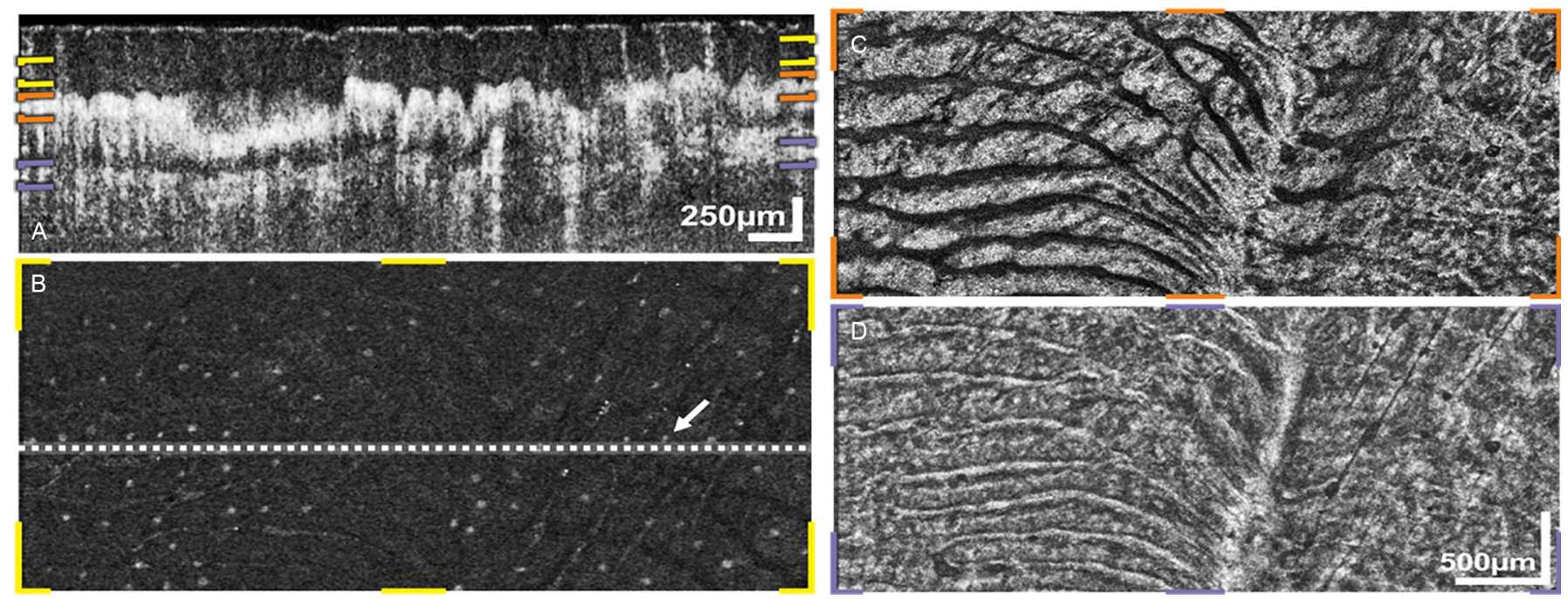

Fig. 12 Images of scar tissue at index finger taken with the 1300-nm partially-integrated SD-OCT system: (A) Cross-section displaying the irregularities caused due to an incision after complete healing. (B, yellow) In the en face view of the region at the layer of the stratum corneum the typical matrix-like distribution of sweat ducts (bright spots) appears on the left and right portion of the image. (C, orange) The tissue distortions become obvious at the layer of the living epidermis and the dermal papillae. (D, violet) Inside the dermis the denser fibrous material of the scar has replaced the normal tissue including the vascular support. The scale bars denote $250 \mu \mathrm{m}$ in cross sections and $500 \mu \mathrm{m}$ in en face sections. 


\section{Conclusions and outlook}

Our results have demonstrated the feasibility and the great potential of on-chip SD-OCT systems. Although spectrometer and MI are the key components of an SD-OCT system to be integrated on a chip, it is necessary to integrate the detector, the light source, and the lenses on the same chip for a fully integrated SD-OCT system. Integration of these components would further decrease the size and increase the stability of OCT systems. However, realization of these components necessitates either a compatible fabrication technique for heterogeneous integration of active (e.g., InP/ InGaAsP) and passive (e.g., SiON) components or accurate and stable wafer bonding techniques (Brouckaert, Roelkens, van Thourhout, \& Baets, 2007; Mathine, 1997; Monat et al., 2001). One possible approach for realizing an integrated light source could be directing the emitted light from active region using $45^{\circ}$ turning mirrors toward the passive layer, which is bonded to the active layer by using the wafer bonding techniques described elsewhere (Roelkens et al., 2006, 2008). In conclusion, considering the current performance of the partially-integrated SD-OCT systems and the potential of integrated optics, in the near future the fully integrated OCT systems will be demonstrated and with their much lower cost, size, and higher stability, they will become accessible to a significantly larger group of applications and users.

\section{References}

Adar, R., Henry, C., Kazarinov, R., Kistler, R., \& Weber, G. (1992). Adiabatic 3-dB couplers, filters, and multiplexers made with silica waveguides on silicon. Journal of Lightwave Technology, 10, 46-50.

Akca, B. I. (2016a). Non-moving scanner design for OCT systems. Optics Express, 24(25), 28459-28466.

Akca, B. I. (2016b). Design of a high-speed multiple reference optical coherence tomography system. Optics Express, 24(23), 26709-26714.

Akca, B. I., Chang, L., Sengo, G., Wörhoff, K., de Ridder, R. M., \& Pollnau, M. (2012). Polarization-independent enhanced-resolution arrayed-waveguide grating used in spectral-domain optical low-coherence reflectometry. IEEE Photonics Technology Letters, 24, 848-850.

Akca, B. I., \& Doerr, C. R. (2019). Interleaved silicon nitride AWG spectrometers. IEEE Photonics Technology Letters, 31, 90-93.

Akca, B. I., Doerr, C. R., Sengo, G., Wörhoff, K., Pollnau, M., \& de Ridder, R. M. (2012). Broad-spectral-range synchronized flat-top arrayed-waveguide grating applied in a 255-channel cascaded spectrometer. Optics Express, 20, 18313-18318.

Akca, B. I., Považay, B., Alex, A., Wörhoff, K., de Ridder, R. M., Drexler, W., et al. (2013). Miniature spectrometer and beam splitter for an optical coherence tomography on a silicon chip. Optics Express, 21(14), 16648-16656. 
Akca, B. I., Weiss, N., Coumans, F., \& van Leeuwen, T. G. (2017). Integrated-optics based multi-beam imaging for speed improvement of OCT systems. In Proc. SPIE 10056, Design and Quality for Biomedical Technologies X, 100560Q.

Akca, B. I., et al. (2012). Toward spectral-domain optical coherence tomography on a chip. IEEE Journal of Selected Topics in Quantum Electroncis, 18, 1223-1233.

Andersen, P. E., Jørgensen, T. M., Thrane, L., Tycho, A., \& Yura, H. T. (2008). Optical coherence tomography: Biological and medical physics, biomedical engineering. Chapter 5 (pp. 73-115).

Avrutsky, I., Chaganti, K., Salakhutdinov, I., \& Auner, G. (2006). Concept of a miniature optical spectrometer using integrated optical and micro-optical components. Applied Optics, 45, 7811.

Bona, G. L., Germann, R., Horst, F., Offrein, B. J., \& Salemink, H. W. M. (1999). Versatile silicon-oxynitride planar lightwave circuits for interconnect applications. In Proc. parallel interconnects (pp. 145-148).

Bourquin, S., Seitz, P., \& Salathé, R. P. (2001). Optical coherence tomography based on a two-dimensional smart detector array. Optics Letters, 26, 512-514.

Bourquin, S., et al. (2005). Parallel broadband fluorescent light source for optical coherence tomography. In Proc. SPIE, coherence domain optical methods and optical coherence tomography in biomedicine IX, 5690, San Jose, CA, USA (pp. 209-213).

Brezinski, M. E., et al. (1996). Imaging of coronary artery microstructure (in vitro) with optical coherence tomography. American Journal of Cardiology, 77, 92-93.

Brouckaert, J., Roelkens, G., van Thourhout, D., \& Baets, R. (2007). Thin-film III-V photodetectors integrated on silicon-on-insulator photonic ICs. Journal of Lightwave Technolog $\gamma, 25,1053-1060$.

Chang, L. (2016). Chip-based common-path swept-source optical coherence tomography system. PhD thesis.

Chang, L., et al. (2016). Chip based common-path optical coherence tomography system with an on-chip microlens and multi-reference suppression algorithm. Optics Express, 24, 12635-12650.

Cheben, P. (2007). Wavelength dispersive planar waveguide device: Echelle gratings and arrayed waveguide gratings. In M. L. Calvo, \& V. Laksminarayanan (Eds.), Optical waveguide: From theory to applied technologies. Taylor \& Francis. Chapter 5.

Cheben, P., et al. (2007). A high-resolution silicon-on-insulator arrayed waveguide grating microspectrometer with sub-micrometer aperture waveguides. Optics Express, 15, 2299-2306.

Cherchi, M. (2003). Wavelength-flattened directional couplers: a geometrical approach. Applied Optics, 42, 7141-7148.

Choi, D., Hiro-Oka, H., Furukawa, H., Yoshimura, R., Nakanishi, M., Shimizu, K., et al. (2008). Fourier domain optical coherence tomography using optical demultiplexers imaging at 60,000,000 lines/s. Optics Letters, 33, 1318-1320.

Chuang, R. W., \& Liao, Z. L. (2009). 2 x 2 thermo-optic silicon oxynitride optical switch based on the integrated multimode interference waveguides. Journal of Electrochemical Society, 157, H149-H152.

Clements, J., Zvyagin, A., Silva, K., Wanner, T., Sampson, D., \& Cowling, W. (2004). Optical coherence tomography as a novel tool for non-destructive measurement of the hull thickness of lupin seeds. Plant Breeding, 123, 266-270.

Culemann, D., Knuettel, A., \& Voges, E. (2000). Integrated optical sensor in glass for optical coherence tomography. IEEE Journal of Selected Topics in Quantum Electronics, 6(5), 730-734.

Doerr, C. R. (2015). Silicon photonic integration in telecommunications. Frontiers in Physics, $3,1-16$.

Germann, R., et al. (2000). Silicon oxynitride layers for optical waveguide applications. Journal of Electrochemical Society, 147, 2237-2241. 
Goldman, D. S., White, P. L., \& Anheier, N. C. (1990). Miniaturized spectrometer employing planar waveguides and grating couplers for chemical analysis. Applied Optics, 29, 4583.

Hammer, D. X. (2011). Advances in retinal imaging. In N. Iftimia, W. R. Brugge, \& D. X. Hammer (Eds.), Advances in optical imaging for clinical medicine (pp. 85-161). NJ, USA: Wiley.

Häusler, G., \& Lindner, M. W. (1998). Coherence radar' and 'spectral radar'-New tools for dermatological diagnosis. Journal of Biomedical Optics, 3, 21-31.

Hu, Z., Pan, Y., \& Rollins, A. M. (2007). Analytical model of spectrometer-based two-beam spectral interferometry. Applied Optics, 46, 8499-8505.

Huang, D., et al. (1991). Optical coherence tomography. Science, 254, 1178-1181.

Jiao, Y., Tilma, B. W., Kotani, J., Nötzel, R., Smit, M. K., He, S., et al. (2012). InAs/InP (100) quantum dot waveguide photodetectors for swept-source optical coherence tomography around $1.7 \mu \mathrm{m}$. Optics Express, 13, 3675-3692.

Jinguji, K., Takato, N., Sugita, A., \& Kawachi, M. (1990). Mach-zehnder interferometer type optical waveguide coupler with wavelength-flattened coupling ratio. Electronics Letter, 26, 1326-1327.

Jung, W., Zhang, J., Chung, J., Wilder-Smith, P., Brenner, M., Nelson, J., et al. (2005). Advances in oral cancer detection using optical coherence tomography. IEEE Journal of Selected Topics in Quantum Electronics, 11, 811-817.

Lee, C., Lee, S.-Y., Kim, J.-Y., Jung, H.-Y., \& Kim, J. (2011). Optical sensing method for screening disease in melon seeds by using optical coherence tomography. Sensors, 11, 9467-9477.

Lim, D., Little, B. E., Lee, K., Morse, M., Fujimoto, H., Haus, H. A., et al. (1999). Micron-sized channel dropping filters using Si waveguide devices. Proceedings of the SPIE, 3847, 65.

Little, B. E., \& Murphy, T. E. (1997). Design rules for maximally flat wavelength-insensitive optical power dividers using Mach-Zehnder structures. IEEE Photonics Technology Letters, 9, 1607-1609.

Louisell, W. H. (1954). Analysis of the single tapered mode coupler. The Bell System Technology Journal, 33, 853-870.

Lu, Z., Yun, H., Wang, Y., Chen, Z., Zhang, F., Jaeger, N. A. F., et al. (2015). Broadband silicon photonic directional coupler using asymmetric-waveguide based phase control. Optics Express, 23, 3795-3808.

Maese-Novo, A., et al. (2013). Wavelength independent multimode interference coupler. Optics Express, 21, 7033-7040.

Margallo-Balbas, E., Pandraud, G., \& French, P. J. (2008). Thermo-optical delay line for miniature optical coherence. In vol. 6847. Tomography in the proceedings of the conference on coherence domain optical methods and optical coherence tomography in biomedicine XII (p. S8470).

Mathine, D. L. (1997). The integration of III-V opto-electronics with silicon circuitry. IEEE Journal of Selected Topics in Quantum Electronics, 3, 952-959.

Monat, C., et al. (2001). InP 2D photonic crystal microlasers on silicon wafer: room temperature operation at $1.55 \mathrm{um}$. Electronics Letters, 37, 764-765.

Nguyen, V. D., et al. (2011). Spectral domain optical coherence tomography imaging with an integrated optics spectrometer. Optics Letters, 36, 1293-1295.

Nguyen, V. D., et al. (2012). Integrated-optics-based swept-source optical coherence tomography. Optics Letters, 37, 4820-4822.

Nikbahkt, H., Karabiyik, M., \& Akca, B. I. (2020). Ultrawide-bandwidth on-chip spectrometer design using novel band-pass filters. Optics Express, 28(15), 23003-23011.

Pitris, C., Brezinski, M. E., Bouma, B. E., Tearney, G. J., Southern, J. F., \& Fujimoto, J. G. (1998). High resolution imaging of the upper respiratory tract with optical coherence tomography. American Journal of Respiratory and Critical Care Medicine, 157, 1640-1644. 
Pitris, C., Goodman, A., Boppart, S. A., Libus, J. J., Fujimoto, J., \& G \& Brezinski M. E. (1999). High-resolution imaging of gynecologic neoplasms using optical coherence tomography. Obstetrics \& Gynecology, 93, 135-139.

Roelkens, G., Brouckaert, J., Van Thourhout, D., Baets, R., Nötzel, R., \& Smit, M. (2006). Adhesive bonding of InP/InGaAsP dies to processed SOI wafers using DVS-bis-BCB. Journal of Electrochemical Society, 153, G1015.

Roelkens, G., et al. (2008). Wafer bonding and heterogeneous integration: Technology and integrated devices (pp. 87-90). Netherlands: ECIO.

Sander, D., Blume, O., \& Müller, J. (1996). Microspectrometer with slab-waveguide transmission gratings. Applied Optics, 35, 4096.

Sander, D., \& Mueller, J. (2001). Selffocussing phase transmission grating for an integrated optical microspectrometer. Sensors and Actuators A, 88, 1.

Smit, M. K., \& van Dam, C. (1996). PHASAR-based WDM-devices: principles, design and applications. IEEE Journal of Selected Topics in Quantum Electronics, 2, 236-250.

Swanson, E. A., Huang, D., Hee, M. R., Fujimoto, J. G., Lin, C. P., \& Puliafito, C. A. (1992). High-speed optical coherence domain reflectometry. Optics Letters, 17, $151-153$.

Takada, K., Abe, M., Shibata, T., \& Okamoto, K. (2001). 10 GHz-spaced 1010-channel tandem AWG filter consisting of one primary and ten secondary AWGs. IEEE Photonics Technology Letters, 13, 577-578.

Takada, K., Abe, M., Shibata, T., \& Okamoto, K. (2002). 1-GHz-spaced 16-channel arrayed-waveguide grating for a wavelength reference standard in DWDM network systems. IEEE Journal of Lightwave Technology, 20, 850-853.

Takada, K., Ade, M., Shibita, T., \& Okamoto, K. (2002). A 25-GHz-spaced 1080-channel tandem multi/demultiplexer covering the $\mathrm{S}_{-}, \mathrm{C}_{-}$, and L-bands using an arrayed-waveguide grating with Gaussian passbands as primary filter. IEEE Photonics Technology Letters, 14, 648-650.

Tearney, G. J., Brezinski, M. E., Southern, J. F., Bouma, B. E., Boppart, S. A., \& Fujimoto, J. G. (1997a). Optical biopsy in human gastrointestinal tissue using optical coherence tomography. American Journal of Gastroenterolgy, 92, 1800-1804.

Tearney, G. J., Brezinski, M. E., Southern, J. F., Bouma, B. E., Boppart, S. A., \& Fujimoto, J. G. (1997b). Optical biopsy in human urologic tissue using optical coherence tomography. Journal of Urology, 157, 1915-1919.

Thomson, D., et al. (2016). Roadmap on silicon photonics. Journal of Optics, 18, 73003.

Tilma, B. W., et al. (2012). Integrated tunable quantum-dot laser for optical coherence tomography in the $1.7 \mu \mathrm{m}$ wavelength region. IEEE Journal of Quantum Electronics, 48, 87-98.

van Leeuwen, T. G., et al. (2018). On-chip Mach-Zehnder interferometer for OCT systems. Advanced Optical Technologies, 7(12), 103-106.

van Wijk, A., Doerr, C. R., Ali, Z., Karabiyik, M., \& Akca, B. I. (2020). Compact ultrabroad-bandwidth cascaded arrayed waveguide gratings. Optics Express, 28(10), 14618-14626.

Verboven, P., et al. (2013). Optical coherence tomography visualizes microstructure of apple peel. Postharvest Biological Technology, 78, 123-132.

Wang, Q., Lu, J., \& He, S. (2002). Optimal design method of a low-loss broadband Y branch with a multimode waveguide section. Applied Optics, 41(36), 7644-7649.

Wörhoff, K., Klein, E. J., Hussein, M. G., \& Driessen, A. (2008). Silicon oxynitride based photonics. In Proceedings of IEEE international conference on transparent optical networks (IEEE) (pp. 266-269).

$\mathrm{Xu}$, D.-X., et al. (2007). High bandwidth SOI photonic wire ring resonators using MMI couplers. Optics Express, 15, 3149-3155.

Youngquist, R. C., Carr, S., \& Davies, D. E. N. (1987). Optical coherence domain reflectometry: a new optical evaluation technique. Optics Letters, 12, 158-160. 
Yun, S. H., Tearney, G. J., Bouma, B. E., Park, B. H., \& de Boer, J. F. (2003). High-speed spectral-domain optical coherence tomography at $1.3 \mu \mathrm{m}$ wavelength. Optics Express, 11 , 3598-3604.

Yurtsever, G., Považay, B., Alex, A., Zabihian, B., Drexler, W., \& Baets, R. (2014). Photonic integrated Mach-Zehnder interferometer with an on-chip reference arm for optical coherence tomography. Biomedical Optics Express, 5, 1050.

Yurtsever, G., Weiss, N., Kalkman, J., van Leeuwen, T. G., \& Baets, R. (2014). Ultracompact silicon photonic integrated interferometer for swept-source optical coherence tomography. Optics Letters, 39(17), 5228-5231. 